\title{
SPECTROMETRIC INVESTIGATION OF CEFPROZIL IN BULK POWDER AND IN PHARMACEUTICAL FORMULATIONS
}

\author{
Sobhy M. El-Adl* and Hanaa M. Saleh \\ Pharmaceutical" and Analytical Chemistry Department, \\ Faculty of Pharmacy, University of Zagazig, Zagazig, Egypt.
}

\begin{abstract}
:
Three accurate methods were developed for the quantitative determination of cefprozil in pure form and in its dosage forms. The first method was based upon the interaction of the drug with 3-methyl-benzothiazolinone-2-hydrazone (MBTH) in the presence of ceric ammonium sulfate or ferric chloride as an oxidizing agent, where the formed color was measured at $\lambda 521 \mathrm{~nm}$ or $624 \mathrm{~nm}$, respectively. The second method was based on the chelate formation with palladium (II) chloride $\left(\mathrm{PdCl}_{2}\right)$ in the presence of buffered medium, where the formed complex was determined at $\lambda 345$ $\mathrm{nm}$. The third method was based upon the reaction of the neutral solution of the hydrolysis product of drug with each of silver nitrate \& lead acetate standard solutions, forming drug-metal complex and the metal ion contents were determined directly or indirectly by atomic absorption spectroscopy (AAS). The reaction conditions of the proposed methods were studied and optimized. The precision of the proposed methods was achieved by determining different samples of bulk powder and pharmaceutical dosage forms. The validity of the methods was assessed by applying the standard addition technique and the results were compared with those obtained by the reference method showing a great agreement.
\end{abstract}

Keywords: Cefprozil; 3-Methyl benzothiazolinone-2-hydrazone; Palladium (II) chloride; Spectrophotometry; Silver \& lead chelation; Atomic absorption spectrometry.

\section{Introduction:}

Cefprozil (5-thia-1-azabicyclo [4.2.0] oct-2-ene-2-carboxylic acid, 7-[amino(4-

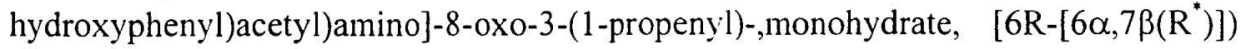
is a semisynthetic broad spectrum oral cephalosporin consisting of $\sim 90: 10 \mathrm{Z} / \mathrm{E}$ isomeric mixture. It is indicated for the treatment of upper \& lower respiratory tract infections, skin infections, and uncomplicated urinary tract infections [1]. There are no published spectrometric methods, until now: for the quantitation of cefprozil. The only reported method for the drug analysis was done by HPLC technique [2,3]. Therefore, it was considered worthwhile to develop a rapid, simple and accurate procedure suitable for application in quality control laboratories. 
The use of MBTH reagent was previously utilized for the analysis of many important drugs [4-8]. Several drugs were determined spectrophotometrically by using palladium (II) chloride as a complexing agent [9-12]. Also, utilization of metal ions in pharmaceutical analysis as reagents for the atomic absorption determination have been described [13-16].

The present paper reports three sensitive and accurate spectrometric methods for the quantitative determination of cefprozil. The proposed methods are based on the reaction of the parent drug with each of 3-methylbenzothiazolinone-2-hydrazone (MBTH) \& palladium (II) chloride and the reaction products are measured spectrophotometrically. Whilst the hydrolysis product of drug is subjected to precipitation with standard solution of silver nitrate \& lead acetate and the metal contents are determined directly or indirectly using atomic absorption spectroscopy (AAS). The optimum conditions are established before the application of the methods to the analysis of the drug as bulk or in pharmaceutical formulations.

\section{Experimental:}

Instrumentation:

1) Shimadzu 260 uv-visible recording spectrophotometer.

2) Shimadzu atomic absorption flame spectrophotometer, model AA-640-13.

3) Chemocadet pH-meter, model 5984-50 (USA).

\section{Materials and reagents:}

All reagents were of analytical grade and all solvents were of spectroscopic grade. Cefprozil was obtained from Bristol-Myers Squibb (Cairo, Egypt). Cefzil tablets were kindly supplied by Bristol-Myers Squibb, Egypt, labeled to contain 500 $\mathrm{mg}$ of anhydrous cefprozil per each tablet. 3-Methylbenzothiazolinone-2-hydrazone (MBTH) was obtained from Aldrich Chem.Co. USA, and prepared as a $5 \times 10^{-3} \mathrm{M}$ solution by dissolving $107 \mathrm{mg}$ in $100 \mathrm{ml} 0.1 \mathrm{M} \mathrm{HCl}$. Ceric ammonium sulfate (Sigma, WI, USA) was prepared as $0.1 \mathrm{M}$ in $0.4 \%$ (v/v) sulfuric acid. Ferric chloride was freshly prepared as $1 \%(\mathrm{w} / \mathrm{v})$ in dist.water. Palladium (II) chloride (Sigma, Milwaukee, WI, USA) was prepared as a $2 \times 10^{-3} \mathrm{M}$ solution by dissolving $35.5 \mathrm{mg}$ in $1 \mathrm{ml}$ of conc. $\mathrm{HCl}$ followed by the addition of $50 \mathrm{ml}$ of boiled water and diluting to $100 \mathrm{ml}$ with water in a volumetric flask. Britton-Robinson buffer solutions [17] of the $\mathrm{pH}$ range (3.5-8.5) were prepared by mixing $0.04 \mathrm{M}$ orthophosphoric, boric and acetic acids with the appropriate volume of $0.4 \mathrm{M}$ sodium hydroxide. The $\mathrm{pH}$ of each buffer solution was determined. Silver nitrate was prepared as $2 \times 10^{-3} \mathrm{M}$ solution (34 $\mathrm{mg} \%$ $\mathrm{w} / \mathrm{v})$. Lead acetate was prepared as $6 \times 10^{-3} \mathrm{M}$ solution $(195 \mathrm{mg} \% \mathrm{w} / \mathrm{v})$. Acetic acid ( $10 \% \mathrm{v} / \mathrm{v}$ solution), Ammonium hydroxide ( $10 \% \mathrm{v} / \mathrm{v}$ solution), and sodium hydroxide (0.5 M solution). Standard solution of the drug was prepared by dissolving the equivalent of $50 \mathrm{mg}$ of cefprozil in distilled water in $100 \mathrm{ml}$ calibrated flask and the volume was completed with the same solvent. 


\section{General procedures:}

\section{1) MBTH-method:}

To a set of $25 \mathrm{ml}$ volumetric flasks, different volumes of the drug standard solution, within the concentration range cited in Table (1), were quantitatively transferred. To each flask, $2 \mathrm{ml}$ ceric ammonium sulfate solution (or $2 \mathrm{ml}$ ferric chloride solution) followed by $2 \mathrm{ml} \mathrm{MBTH}$ reagent were added. The reaction mixtures were left to stand at room temperature $\left(25 \pm 1^{\circ} \mathrm{C}\right)$ for 20 minutes. The flasks were then completed to volume with distilled water and the absorbance of the resulting solutions was measured at $521 \mathrm{~nm}$ or $624 \mathrm{~nm}$ against the corresponding reagent blank.

\section{2) Palladium (II) chloride method:}

To a set of $25 \mathrm{ml}$ volumetric flasks, various volumes of the drug standard solution, within the concentration range cited in Table (1), were pipetted. To each flask, $1 \mathrm{ml}$ of Britton-Robinson buffer solution ( $\mathrm{pH}$ 8) followed by $3 \mathrm{ml} \mathrm{PdCl}_{2}$ solution were added. The reaction mixtures were left to stand at room temperature $\left(25 \pm 1{ }^{\circ} \mathrm{C}\right)$ for 5 minutes. The flasks were then completed to volume with distilled water and the absorbance of the resulting solutions was measured at $345 \mathrm{~nm}$ against the corresponding reagent blank.

\section{3) Atomic absorption spectrometric method (AAS):}

Appropriate volumes of the drug standard solution, within the concentration range cited in Table (1), were transferred. Then, $(0.5-2 \mathrm{ml})$ of $0.5 \mathrm{M}$ sodium hydroxide solution was added and the solution was heated in a water bath at $90^{\circ} \mathrm{C}$ for 20 minutes. The hydrolyzed drug solution was cooled and neutralized with $10 \%(\mathrm{v} / \mathrm{v})$ acetic acid solution. To two sets of flasks, a double volume of each of silver nitrate and lead acetate solutions were added. The mixture was protected from light by wrapping the flasks with aluminum foil (in case of silver), shaken and filtered through Whatman No. 44 filter papers. The precipitates were washed with bidistilled water until silver and lead ions free.

a) Direct method: The obtained precipitate was dissolved in the least amount of dilute ammonium (in case of silver) or dilute acetic acid (in case of lead), and completed to $100-\mathrm{ml}$ with bidistilled water in the calibrated flasks.

b) Indirect method: The filtrates and washings were collected in 100-ml calibrated flasks and completed to volume with bidistilled water.

A blank (omitting the addition of the drug) was performed; the solutions ( $a, b)$ were aspirated into an oxidizing air acetylene flame; and the concentration of the consumed and the residual $\left(\mathrm{Ag}^{+} \& \mathrm{~Pb}^{2+}\right)$ were calculated from the calibration graph of standard silver nitrate and lead acetate solutions, respectively. The absorbance was measured at the following conditions: line wavelength $\left(2833 \AA\right.$ for $\mathrm{Pb}^{2+} \& 3281 \AA$ for $\mathrm{Ag}^{+}$), lamp current $7 \mathrm{~mA}$, slit width $3.8 \AA$, air pressure $10 \mathrm{~min}^{-1}$ and acetylene pressure $2.61 \mathrm{~min}^{-1}$. 


\section{4) Procedure for the commercial tablets:}

Twenty tablets were weighed and powdered. A quantity of the powder equivalent to $50 \mathrm{mg}$ of cefprozil was weighed and transferred to a $100 \mathrm{ml}$ calibrated flask with the aid of about $50 \mathrm{ml}$ distilled water. The flask was sonicated for 15 minutes in an ultrasonic water bath. The flask was completed to volume with the same solvent and filtered. Aliquots from the filtrate were used for the application of the suggested general procedures.

\section{RESULTS AND DISCUSSION}

\section{1) MBTH-method:}

It has been reported that MBTH loses a proton and two electrons to form the electrophilic intermediate which is the active coupling species. This intermediate undergoes electrophilic substitution with phenols in the ortho and para-positions to produce the colored product [18]. Ceric ammonium sulfate or ferric chloride acts as an oxidizing agent converting MBTH into the form I which couples with cefprozil to form the colored product II. The mechanism of the reaction may interpreted according to the following scheme:
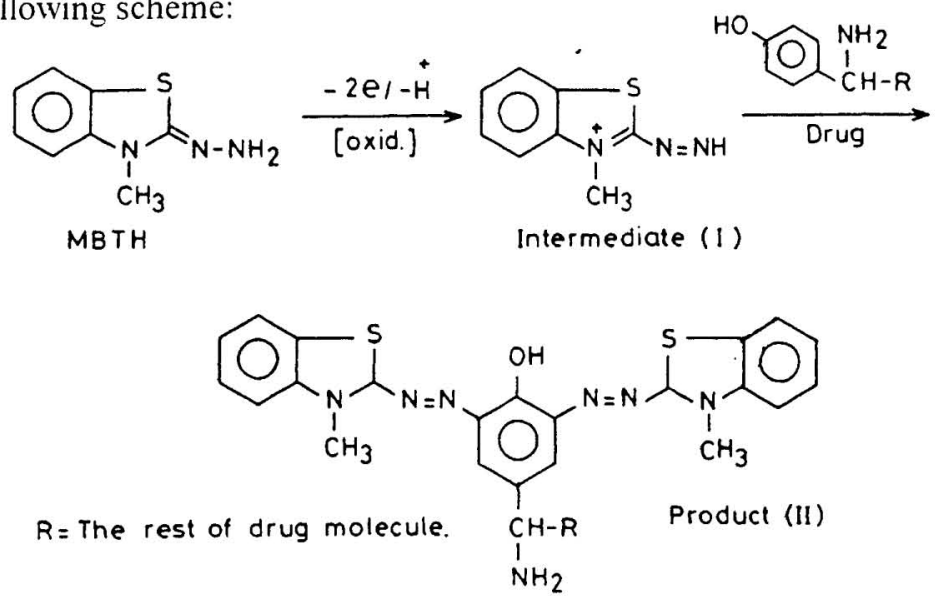

Scheme (1): Suggested reaction pathway of cefprozil with MBTH reagent.

The conditions for the production of the most intense and stable color were studied. The investigated oxidizing agents were ceric ammonium sulfate and ferric chloride. On using the former oxidizer, it gave product with maximum absorption at $521 \mathrm{~nm}$, while on using the later oxidizer, it gave product with two absorption maxima at $624 \& 660 \mathrm{~nm}$ Fig. (1). Cefprozil itself has two absorption maxima in the UV region ( $\mathrm{pH} 7$ phosphate buffer) at $228 ; 279 \mathrm{~nm}$, while MBTH reagent has $\lambda_{\max }$ at $218 \mathrm{~nm}$ in an aqueous medium. The color product gave maximum intensity on using 3 $\mathrm{ml}$ of MBTH reagent and when the reaction was left for 20 minutes at room temperature indicating that the reaction was completed after this time. The absorbance of the color was found to be stable for at least 6 hours. 


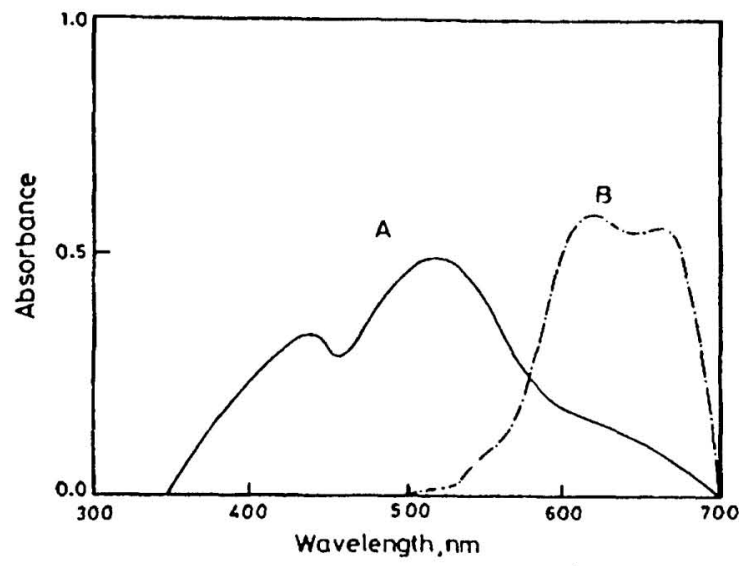

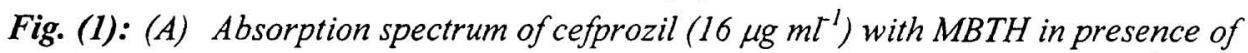

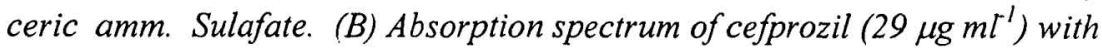
$M B T H$ in presence of ferric chloride.

Stoichiometric relationship of the reactants (drug: MBTH) was found to be $1: 2$ using the continuous variation method $[19,20]$ Fig. (3). This may be explained as the drug has two free ortho positions (Scheme I). Under the described experimental conditions, a linear correlation was obtained between absorbance, $\mathrm{A}$ and the concentration, $\mathrm{C}$, of cefprozil over the range of $6-36 \mu \mathrm{g} \mathrm{ml}^{-1}$ in the final measured solution. The linear regression equation, molar absorptivity, and Sandell's sensitivity were summarized with the other data in Table (1).

Table (1): Quantitative parameters for the proposed spectrometric determination of cefprozil.

\begin{tabular}{|c|c|c|c|c|c|c|c|}
\hline \multirow{3}{*}{ Parameters } & \multicolumn{3}{|c|}{ Spectrophotometric } & \multicolumn{4}{|c|}{ Atomic absorption (AAS) } \\
\hline & \multirow[t]{2}{*}{$\mathrm{PdCl}_{2}$} & \multicolumn{2}{|c|}{ MBTH } & \multicolumn{2}{|c|}{ Using $\mathrm{Ag}^{+}$} & \multicolumn{2}{|c|}{ Using $\mathrm{Pb}^{2+}$} \\
\hline & & Using $\mathrm{Ce}^{4+}$ & Using $\mathrm{Fe}^{3+}$ & Direct & Indirect & Direct & Indirect \\
\hline $\begin{array}{l}\text { Beer's law limit } \\
(\mu \mathrm{g} / \mathrm{ml})\end{array}$ & $9-45$ & $6-36$ & $6-36$ & $2-10$ & $2-10$ & $10-50$ & $10-50$ \\
\hline $\begin{array}{l}\text { Molar absorptivity } \\
\left(1 . \mathrm{mole}^{-1} \mathrm{~cm}^{-1}\right)\end{array}$ & $0.67 \times 10^{4}$ & $1.17 \times 10^{4}$ & $0.79 \times \times 10^{4}$ & $1.85 \times 10^{6}$ & $2.05 \times 10^{6}$ & $5.23 \times 10^{5}$ & $6.12 \times 10^{5}$ \\
\hline $\begin{array}{l}\text { Sandell's sensitivity } \\
\left(\mu \mathrm{g} \mathrm{cm}^{-2}\right) \\
\text { Regression equation* }\end{array}$ & 0.058 & 0.033 & 0.049 & 0.000058 & 0.000052 & 0.00039 & 0.00034 \\
\hline Intercept (a) & 0.026 & 0.018 & 0.006 & -0.18 & 0.59 & 0.06 & -0.05 \\
\hline Slope (b) & 0.016 & 0.029 & 0.020 & 15.49 & 17.12 & 2.44 & 2.86 \\
\hline Correl. Coeff. (r) & 0.9996 & 0.9998 & 0.9997 & 1.00 & 0.9995 & 1.00 & 0.9993 \\
\hline
\end{tabular}

${ }^{*} \mathrm{~A}=\mathrm{a}+\mathrm{bC}$ where $(\mathrm{A})$ is the absorbance and $(\mathrm{C})$ is the concentration in $\mu \mathrm{g} \cdot \mathrm{ml}^{-1}$. 


\section{2) Palladium (II) chloride method:}

Palladium (II), as one of the transition elements was found to form stable complexes with many ligands containing heteroatoms [21]. Addition of $\mathrm{PdCl}_{2}$ to cefprozil produced yellow complex that was soluble in Britton-Robinson buffer solutions in the $\mathrm{pH}$ range of (5.5-8.5). The resulting chelate is possibly due to the complexation of Palladium (II) ion and the sulfur atom of the $\beta$-lactam ring of cefprozil. Palladium (II) chloride reagent in an aqueous medium has two absorption maxima at $236.5 ; 206.5 \mathrm{~nm}$. The complex gave an absorption peak at $345 \mathrm{~nm}$ (Fig. 2) which was therefore used for the analytical determination.

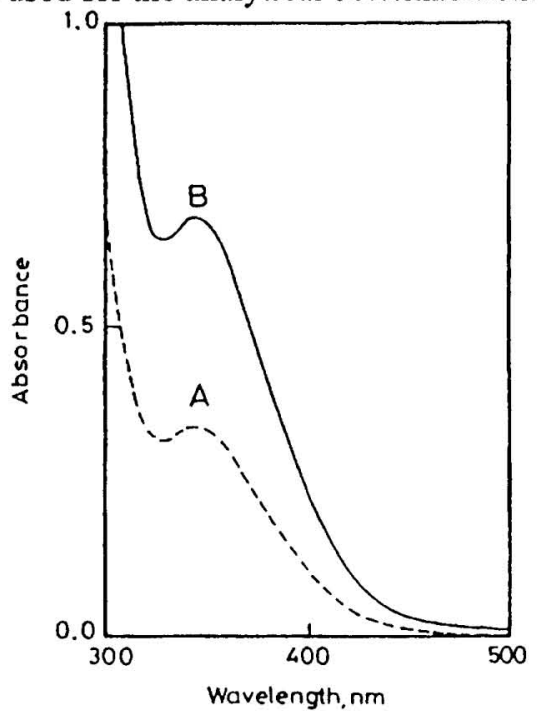

Fig. (2): Absorption spectra of cefprozil with $\mathrm{PdCl}_{2}$ in presence of the buffer, using

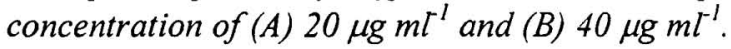

The absorption maximum, and the intensity of the complex produced were not greatly influenced by the change of the $\mathrm{pH}$ of the medium, indicating that only one type of complex was produced. The maximum sensitivity was obtained when a medium containing $1 \mathrm{ml}$ of the Britton-Robinson buffer of $\mathrm{pH} 8$ and $3 \mathrm{ml}$ of $\mathrm{PdCl}_{2}$ solution were used. No effect was observed neither on using the anionic surfactant sodium lauryl sulfate (SLS) nor on raising the temperature from room temperature $\left(25^{\circ} \mathrm{C}\right)$ into $70^{\circ} \mathrm{C}$. Full color development was achieved through 5 minutes and the absorbance remained constant for at least 1 hour.

The composition of the complex was established by the equimolar solution Job's continuous variation method indicating the formation of $1: 1$ cefprozil-Palladium (II) complex, Fig. (3). Under the described experimental conditions. a linear relationship was obtained for the absorbance of the complex when the concentration of the drug was in range of $9-45 \mu \mathrm{g} \cdot \mathrm{ml}^{-1}$ in the final measured solution. The regression equation describing the calibration graph, with the intercept(a), slope (b), correlation coefficient(r), the molar absorptivity( $(\varepsilon)$ were also shown in Table (1). 


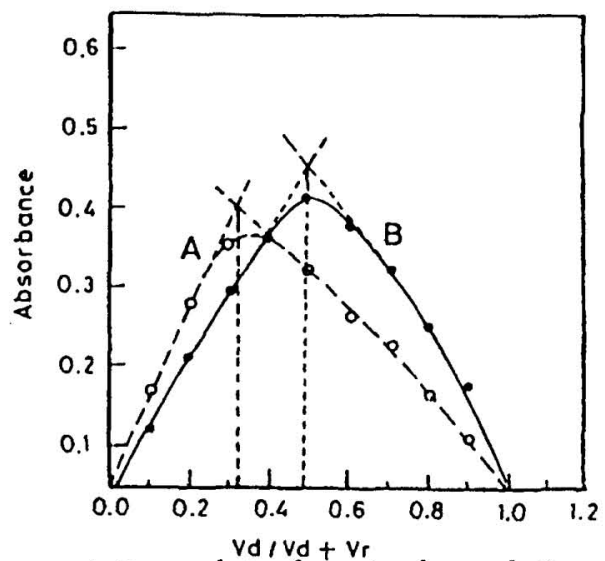

Fig. (3): Continuous variation plot of equimolar solutions of cefprozil and the reagent $\left(1.5 \times 10^{-3} \mathrm{M}\right)$; (A) $\mathrm{MBTH}$ reagent and (B) $\mathrm{PdCl}_{2}$ reagent.

\section{3) Atomic absorption methods: (AAS)}

The neutral solutions of the alkaline hydrolysis produced of cefprozil were found to give brown precipitate with both of silver nitrate and lead acetate solutions. These precipitates were the basis for the micro-determination of the drug. Both of the metal ions could be estimated directly (in the precipitate) or indirectly (in the filtrate) by the atomic absorption spectrometry (AAS). The expected reaction mechanism may be represented by the following scheme:

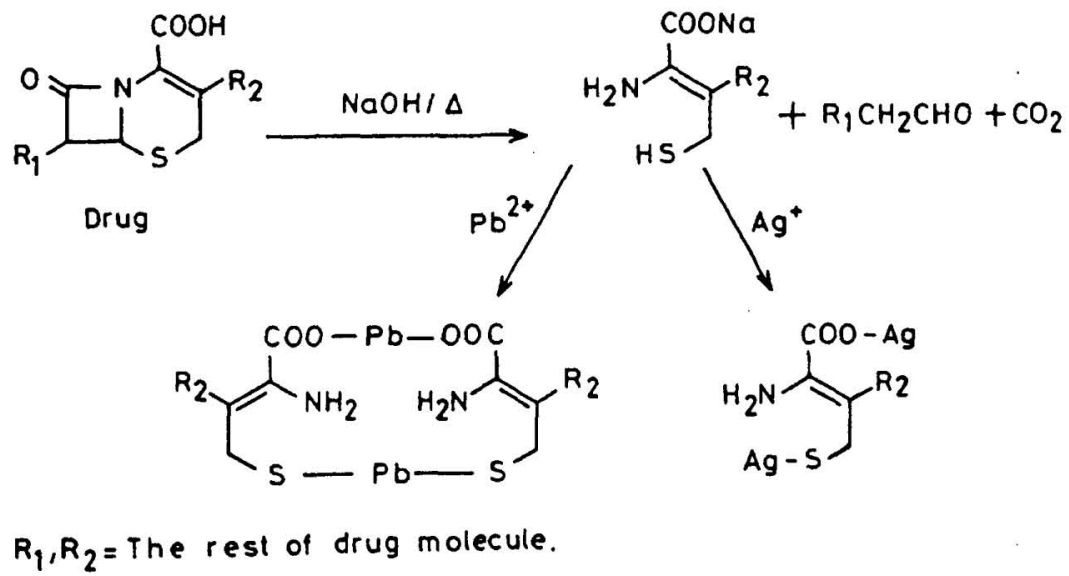

Scheme (2): Suggested reaction pathway of alkaline-degradation product of cefprozil with both silver and lead ions.

Different factors affecting the reaction were carefully studied. It was found that $0.5 \mathrm{ml}$ of $0.5 \mathrm{M} \mathrm{NaOH}$ solution was sufficient for complete hydrolysis of cefprozil for the procedure using $\mathrm{Ag}(\mathrm{I})$ ion, but this concentration wasn't enough for the procedure 
using $\mathrm{Pb}(\mathrm{II})$ ion, that may be due to the relative high concentration of drug used in this procedure (about five times more than that used in the $\mathrm{Ag}(\mathrm{I})$ ion procedure). In this procedure $2 \mathrm{ml}$ of $0.5 \mathrm{M} \mathrm{NaOH}$ solution was used for the hydrolysis step. Heating at $90^{\circ} \mathrm{C}$ for 20 minutes in presence of $\mathrm{NaOH}$ was essential for the complete cefprozil hydrolysis.

The precipitation process was carried out in a neutral aqueous media. The acidic medium has a solubilizing effect on the precipitate, while the alkaline medium precipitates the metal ions as oxide or hydroxide leading to higher results for the direct procedure. For the complete precipitation of the drug-degradation product, double amount of silver nitrate or lead acetate standard solution should be added. Excessive reagents must be avoided due to their solubilizing effect on the formed precipitate. Complete precipitation was found to occur instantaneously and the absorbance was stable for 24 hours. The stoichiometric relationship indicated a molar ratio of 1:1 and 1:2 cefprozil to lead and silver ions, respectively, Scheme (2).

The concentration of metal ions were calculated from the corresponding calibration graph with the same background composition or from the general regression equation, Table (1). Where each $1 \mathrm{mg}$ of $\mathrm{Ag}^{+}$ion is equivalent to $1.80 \mathrm{mg}$ of cefprozil, and each $1 \mathrm{mg}$ of $\mathrm{Pb}^{2+}$ ion is equivalent to $1.88 \mathrm{mg}$ of the drug.

\section{Quantification, accuracy and precision:}

The validity of the proposed procedures for the quantitative determination of cefprozil was assessed by applying the methods to the commercial preparations (tablets). Recoveries were determined by adding standard drug to the pre-analyzed tablets followed by its estimation using standard addition technique. The obtained results were reproducible with low standard deviation on estimating five replicates of the drug within Beer's law limits. Evaluation of the proposed methods was performed by the statistical analysis of the data, where intercepts, slopes, correlation coefficients, detection limits and molar absorptivities are shown in Table (1).

There is no interferences was observed in the determination of cefprozil from the excipients such as glucose, lactose, starch, citric acid, saccharin, gum acacia and magnesium stearate, when present in 120 fold molar excess. Also, there was no interferences from the thermal and hydrolytic degradation products of the drug.

The performance of the suggested methods was checked by calculation of the student $t$ and variance $F$-values compared with the reference spectrophotometric method $\left(\mathrm{A}_{\max }\right.$ method) [22,23]. Tables $(2,3)$. These results indicate that the suggested methods are accurate, precise and show no significant difference between them and the reference method. 
Table (2): Quantitative determination of cefprozil by the proposed methods compared with the reference method.

\begin{tabular}{|c|c|c|c|c|c|c|c|c|}
\hline \multirow{3}{*}{ Parameters } & \multirow{3}{*}{$\begin{array}{l}\text { Refer. } \\
\text { method }\end{array}$} & \multicolumn{3}{|c|}{ Spectrophotometric } & \multicolumn{4}{|c|}{ Atomic absorption (AAS) } \\
\hline & & \multirow[t]{2}{*}{$\mathbf{P d C l}_{2}$} & \multicolumn{2}{|c|}{ MBTH } & \multicolumn{2}{|c|}{ Using $\mathrm{Ag}^{+}$} & \multicolumn{2}{|c|}{ Using $\mathbf{P b}^{2+}$} \\
\hline & & & Using $\mathrm{Ce}^{4+}$ & Using $\mathrm{Fe}^{3+}$ & Direct & Indirect & Direct & Indirect \\
\hline Mean recovery & 99.64 & 99.72 & 99.68 & 99.70 & 99.52 & 99.46 & 99.58 & 99.62 \\
\hline Variance & 0.185 & 0.172 & 0.227 & 0.265 & 0.407 & 0.462 & 0.277 & 0.425 \\
\hline SD & 0.430 & 0.415 & 0.476 & 0.515 & 0.638 & 0.680 & 0.526 & 0.652 \\
\hline RSD (\%) & 0.431 & 0.416 & 0.477 & 0.516 & 0.641 & 0.683 & 0.528 & 0.654 \\
\hline SE & 0.193 & 0.186 & 0.213 & 0.231 & 0.286 & 0.305 & 0.236 & 0.292 \\
\hline t-test & & 0.30 & 0.14 & 0.20 & 0.34 & 0.50 & 0.19 & 0.06 \\
\hline F-test & & 1.07 & 1.23 & 1.43 & 2.20 & 2.50 & 1.50 & 2.30 \\
\hline
\end{tabular}

- Number of experiments $=5$; where each result is the average of three measurements.

- The tabulated values of $t$ and $F($ at $P=0.05$ ) are 2.31 and 6.39 , respectively.

Table (3): Quantitative determination of cefprozil (in cefzil tablets) using the proposed methods compared with the reference method.

\begin{tabular}{|l|c|c|c|c|c|c|c|c|}
\hline \multirow{2}{*}{ Parameters } & \multirow{2}{*}{$\begin{array}{c}\text { Refer. } \\
\text { method }\end{array}$} & \multicolumn{3}{|c|}{ Spectrophotometric } & \multicolumn{4}{c|}{ Atomic absorption (AAS) } \\
\cline { 3 - 9 } & & PdCl $_{2}$ & \multicolumn{2}{|c|}{ MBTH } & \multicolumn{2}{c|}{ Using $\mathrm{Ag}^{+}$} & \multicolumn{2}{c|}{ Using $\mathrm{Pb}^{2+}$} \\
\cline { 3 - 9 } & & & Using Ce & Using Fe & Direct & Indirect & Direct & Indirect \\
\hline Mean recovery & 99.92 & 99.80 & 99.86 & 99.88 & 100.20 & 100.32 & 100.14 & 100.10 \\
Variance & 0.490 & 0.285 & 0.315 & 0.337 & 0.435 & 0.445 & 0.492 & 0.395 \\
SD & 0.70 & 0.534 & 0.561 & 0.580 & 0.660 & 0.670 & 0.70 & 0.630 \\
RSD (\%) & 0.70 & 0.535 & 0.562 & 0.58 & 0.658 & 0.667 & 0.699 & 0.629 \\
SE & 0.314 & 0.240 & 0.251 & 0.260 & 0.296 & 0.30 & 0.314 & 0.283 \\
t-test & & 0.30 & 0.15 & 0.10 & 0.64 & 0.92 & 0.49 & 0.42 \\
F-test & & 1.72 & 1.56 & 1.45 & 1.13 & 1.10 & 1.00 & 1.24 \\
\hline
\end{tabular}

- Number of experiments $=5$; where each result is the average of three measurements.

- The tabulated values of $t$ and $F($ at $P=0.05$ ) are 2.31 and 6.39 , respectively.

\section{Conclusion:}

Hitherto, there are no data on assay of cefprozil except only one dealing with HPLC method, that requires sephisticated instrument and expensive reagents. Therefore, it was considered worthwhile to develop a rapid, simple and accurate method suitable for the routine analysis of cefprozil in the bulk powder and in the different pharmaceutical formulations. The proposed methods include two sensitive spectrophotometric methods using MBTH \& palladium (II) chloride reagents. In addition to other two atomic absorption spectrometric procedures using silver and lead ions. The statistical analysis of the results confirmed that the developed methods have good accuracy and high precision. The produced color reactions are developed at once 
S. M. EI - A d I and H. M. S a I e h : Spectrometric Investigation of Cefprozil in ...

and are long lasting. Moreover, these methods are very sensitive and can be successfully applied in the quality control of the drug.

\section{References}

[1] Martindale (1999), The complete drug reference, Kathleen Parfitt Editor, 32th Edition, The Pharmaceutical Press, London, p. 172.

[2] Shyu W.C., Shukla U.A., Shah V.R., Papp E.A., Barbhaiya R.H. (1991), Pharm. Res. 8 (8): 992.

[3] The United States Pharmacopoeia (2000), 24, NF 19, p. 348.

[4] Geeta N., Baggi T.R. (1989), Microchem. J. 39: 137.

[5] Sastry C.S.P., Tipirneni A.S.R., Suryanarayana M.V. (1990), J. Pharm. Biomed. Anal. 8 (3): 287.

[6] Devani M.B., Pandya S.S., Shah S.A. (1991), Indian J. Pharm. Sci. 5 (6): 96.

[7] El-Yazbi F.A., Galal S.M., Blaih S.M. (1993), Bull. Soc. Chim. Belg. 102: 233.

[8] Sastry C.S.P., Krishna D.M. (1995), Anal. Lett. 28 (7): 1197.

[9] Jovanovic T.,Stanovic B., Koricanac Z.(1995),J.Pharm. Biomed. Anal. 13(3): 213.

[10] El-Walily A.M., Belal S.F., Bakry R.S. (1996), Ibid. 14: 561.

[11] Stankovic B., Jovanovic T., Masic S., Koricanac Z. (1996), Farmaco 51: 679.

[12] El-Walily A.M., Gazy A.A., Belal S.F., Khamis E.F. (2000), J. Pharm. Biomed. Anal. 22: 385.

[13] Ayad M.M., Khayyal S., Farag N. (1985), Spectrochim. Acta 40B (8): 1127.

[14] Chakraborty D., Das A.K. (1998), At. Spectrosc. 9 (4): 115.

[15] Yebra M.C., Gallego M., Valcarcel M. (1995), Anal. Chim. Acta. 308: 275.

[16] Ayad M.M., Shalaby A.A., Abdellatif H.E., Elsaid H.M. (1999), J. Pharm. Biomed. Anal. 18: 975.

[17] Coch-Frugoni J.A. (1957), Gazz. Chim. Ital. 87: 403.

[18] Sawicki E., Stanley T.W., Hauser T.R., Elbert W., Noe J.L. (1961), Anal. Chem. 33: 722.

[19] Rose J. (1964), Advanced Physico-Chemical Experiments, Pitman, London p.54.

[20] Sawyer D.T., Heineman W.R., Beebe J.M. (1984), Chemistry Experiments for Instrumental Methods, Wiley, New York, p. 198.

[21] Jovanovic T.S., Stankovic B.S. (1989), Analyst 114: 401.

[22] The Merck Index (1996), An Encyclopedia of Chemicals, Drugs and Biologicals, Merck Co. Inc., Twelfth Edition, p. 322.

[23] Miller J.C., Miller J.N. (1993), Statisticals in Analytical Chemistry, Eillis Horwood, Chichester, 3-rd Edition. 